\title{
Intrinsic Optical Signals in Rat Neocortical Slices Measured with Near-Infrared Dark-Field Microscopy Reveal Changes in Extracellular Space
}

\author{
Knut Holthoff and Otto W. Witte \\ Neurologische Klinik der Heinrich Heine Universität, D-40225 Düsseldorf, Germany
}

In the last decade, the measurement of activity-dependent intrinsic optical signals (IOSs) in excitable tissues has become a useful tool for collecting data about spatial patterns of information processing in mammalian brain and spread of excitation. Although the extent of the IOS correlates well with the extent of electrical excitation, its time course is much slower, suggesting that it does not directly monitor the electrical activity. The aim of this study was to investigate the mechanisms responsible for generation of lOSs.

Coronal neocortical brain slices of juvenile rats were electrically stimulated at the border of layer VI and the white matter. The induced columnar-shaped IOSs were recorded using darkfield video microscopy. At corresponding locations, alterations in extracellular $\mathrm{K}^{+}$concentration and extracellular space (ECS) volume were registered using ion-selective microelectrodes. After stimulation, a transient increase of extracellular $\mathrm{K}^{+}$concentration up to $10 \mathrm{~mm}$ and a transient decrease of ECS volume by $\sim 4 \%$ could be observed. The comparison of the time courses of these parameters yielded considerable differences between extracellular $\mathrm{K}^{+}$concentration increase and IOS, but obvious similarities between alterations in ECS volume and IOS. To test the hypothesis that changes in IOS reflect changes in ECS, but not extracellular $\mathrm{K}^{+}$concentration, we recorded under conditions that are known to prevent activity-induced changes in ECS, i.e., in low $\mathrm{Cl}^{-}$solutions and in the presence of furosemide. Both treatments similarly decreased stimulationinduced IOSs and alterations of ECS. However, the effect of these treatments on changes of extracellular $\mathrm{K}^{+}$was different and did not correspond to the changes of IOS.

We conclude that activity-dependent IOSs in rat neocortical slices measured by near-infrared video microscopy reveal changes in ECS. Furthermore, the pharmacological and ion substitutional experiments make it likely that activity-induced IOSs are attributable to cell swelling via a net $\mathrm{KCl}$ uptake and a concomitant water influx.

Key words: intrinsic optical signal; extracellular space; neocortical slice; rat; in vitro; cell-volume changes
Activity-dependent changes of optical properties in excitable tissues were first described several decades ago (Hill and Keynes, 1949). Such activity-dependent intrinsic optical signals (IOSs) after electrical stimulation were observed in different preparations in vitro, such as crab nerves (Hill and Keynes, 1949), cerebral cortical slices (Lipton, 1973; MacVicar and Hochman, 1991; Holthoff et al., 1994), or isolated whole brain (Federico et al., 1994), and in vivo in both animals (Grinvald et al., 1986) and humans (Haglund et al., 1992). Several studies have indicated that the IOSs can serve as a means to monitor the spatial extent of the electrical activity. Amplitudes of electrical signals and IOSs show a good spatial correlation (Gochin et al., 1992; Federico et al., 1994; Holthoff et al., 1994).

In vivo, three components of IOSs can be distinguished, each of which has a maximum at different wavelength. Two of these components can be attributed either to changes in blood volume or to changes in oxygen consumption (Frostig et al., 1990). The origin of the third component is not yet clear. This latter component is wavelength-independent and based on scattered light.

In vitro, only the third component of the IOSs can be observed, which recent studies suggest is attributable to cell swelling (MacVicar and Hochman, 1991; McManus et al., 1993; Andrew

\footnotetext{
Received Sept. 26, 1995; revised Jan. 22, 1996; accepted Jan. 25, 1996.

This work was supported by Sonderforschungsbereich 194 B2.

Correspondence should be addressed to Dr. Otto W. Witte, Neurologische Klinik, Heinrich Heine Universität, Moorenstrasse 5, D-40225 Düsseldorf, Germany. Copyright (c) 1996 Society for Neuroscience $0270-6474 / 96 / 162740-10 \$ 05.00 / 0$
}

and MacVicar, 1994). In hippocampal slices, activity-dependent IOSs, which were measured as changes in light transmission, could be diminished by treatment with furosemide or by reduction of extracellular $\mathrm{Cl}^{-}$without influence on evoked field potential amplitude (MacVicar and Hochman, 1991). In the same preparation, treatment with hypo-osmolar solutions induced IOSs. These IOSs were associated with increased population-spike amplitudes, which were evoked by electrical stimulation and interpreted as measures for extracellular space (ECS) volume (Andrew and MacVicar, 1994). Thus, IOSs in brain slices may be a useful means to monitor changes of the extracellular space with good time and space resolution. Previously, such measurements were only made possible by monitoring changes of the tissue impedance or by monitoring the concentration of certain ions in the extracellular space, which are not membrane-permeable. The aim of the present study was to investigate whether there is a correlation between IOS and ECS volume. The IOS, as monitored by nearinfrared dark-field microscopy, was coregistered with the extracellular potassium concentration and with the concentration of the membrane-impermeable tetramethylammonium (TMA) ions. Additionally, ion substitutional and pharmacological experiments were used.

These experiments demonstrated that the IOSs in vitro correlated tightly with changes in ECS volume and suggest that the changes in ECS volume are primarily because of clearance of $\mathrm{K}^{+}$ from the extracellular space by furosemide-sensitive uptake of $\mathrm{KCl}$ into glial cells. 


\section{MATERIALS AND METHODS}

Juvenile male Wistar rats (14 d old) were anesthetized and decapitated. Brains were rapidly removed and cooled to $4^{\circ} \mathrm{C}$. Using a vibratome, 400 $\mu \mathrm{m}$ thick neocortical coronal slices were prepared and stored at room temperature in artificial CSF (aCSF) containing (in $\mathrm{mM}$ ): $\mathrm{NaCl} 124$, $\mathrm{NaHCO}_{3} 26, \mathrm{KCl} 3, \mathrm{CaCl}_{2} 2, \mathrm{MgSO}_{4} 2, \mathrm{NaH}_{2} \mathrm{PO}_{4} 1.25$, and glucose 10 , equilibrated with $95 \% \mathrm{O}_{2}$ and $5 \% \mathrm{CO}_{2}, \mathrm{pH} 7.4$. Experiments were done on 26 slices from 15 animals. Number of experiments refers to number of slices. In aCSF containing tetramethylammoniumchloride (TMA-Cl), equimolar amounts of $\mathrm{NaCl}$ were omitted. Furosemide (Sigma, St. Louis, MO) was dissolved in aCSF using an ultrasonic bath. The slices were stored in the recording chamber submerged at $32^{\circ} \mathrm{C}$.

To avoid changing light reflections at the boundary between water and air, we covered the recording chamber with a small coverslip. An upright microscope (Axioskop FS, Zeiss, Thornwood, NY) with a $2.5 \times$ Neofluar objective ( 0.075 numerical aperture) was used. The slices were illuminated using a dark-field configuration. The illumination light was filtered with a bandpass interference filter $(750 \pm 50 \mathrm{~nm}$, Oriel). We chose near-infrared light because it penetrates better into the depth of the slice than visible light, resulting in a more homogeneous background intensity distribution of the original microscopic image. The video imaging system consisted of a charge-coupled device (CCD) camera (C 2400-77 type 01, Hamamatsu), a camera control unit including a shading correction system, a video processing unit (DVS 3000, Hamamatsu), and an S-VHS video recorder (RTV 950 PC, Blaupunkt). Experiments were analyzed off-line using a Macintosh FX personal computer equipped with a frame grabber card and National Institutes of Health Image software.

For stimulation (pulses of $200 \mu \mathrm{sec} / 4-9 \mathrm{~V}$ in a train of $50 \mathrm{~Hz}$ for $2 \mathrm{sec}$ ), a bipolar concentric stimulation electrode was placed perpendicular to the surface of the slice at the border of layer VI and the white matter. To detect stimulation-induced changes in IOSs, analog contrast enhancement, background subtraction, and an eightfold digital enhancement were applied on-line to the video images obtained from the CCD camera (Holthoff et al., 1994). Finally, a moving average filter, processing two images at a time, was applied on-line to the video signal to reduce noise. The intensity of IOSs was measured in layer II/III by averaging the pixel intensities of a circle-shaped area $(80 \mu \mathrm{m}$ diameter $)$. To exclude artifacts resulting from changes of light scattering in the whole slice (e.g., because of small movements of the slice), we calculated the difference between optical intensity in the region of interest and a noninvolved reference region. For determination of the time course, 10 images of the IOSs per second were evaluated off-line.

Double-barreled ion-selective microelectrodes (ISMEs) were used to determine extracellular $\mathrm{K}^{+}$concentration and changes in ECS volume. In these experiments, ion concentrations and IOSs were recorded simultaneously. ISMEs were prepared with a liquid potassium ion exchanger (Corning 477317) and calibrated in the conventional manner. In addition to its selectivity for potassium ions, the ion exchanger is highly selective for quarternary ammonium ions (Hansen and Olsen, 1980; Huang and Karwoski, 1992). Changes in ECS volume can be detected by adding 10 mM TMA to extracellular fluid (Ransom et al., 1985; Huang and Karwoski, 1992). To maintain ionic strength, equimolar amounts of $\mathrm{NaCl}$ were omitted. Because of its size and charge, TMA is largely restricted to the extracellular compartment (Nicholson and Phillips, 1981), although it may be rapidly taken up by glial cells to a steady intracellular level (Ballanyi et al., 1990). Changes in extracellular TMA concentration can be interpreted as alterations in ECS volume. Relative alterations in ECS volume were calculated using the following expression (Dietzel et al., 1980):

percentage of shrinkage of $\mathrm{ECS}=\left(1-\frac{\left[\mathrm{TMA}^{+}\right]_{o} \text { before activity }}{\left[\mathrm{TMA}^{+}\right]_{o} \text { after activity }}\right) \times 100$.

In the presence of quarternary ammonium ions, the used ISMEs are virtually blind to potassium ions (Huang and Karwoski, 1992).

\section{RESULTS}

After stimulation at the border of layer VI and the white matter, columnar-shaped IOSs could be detected throughout cortical layers II-VI (Fig. 1A). Layer I was never involved. The induction of IOSs by electrical stimulation was dependent on synaptic transmission. In aCSF containing $4 \mathrm{~mm} \mathrm{Mg}^{2+}$ and no $\mathrm{Ca}^{2+}$, synaptic
A
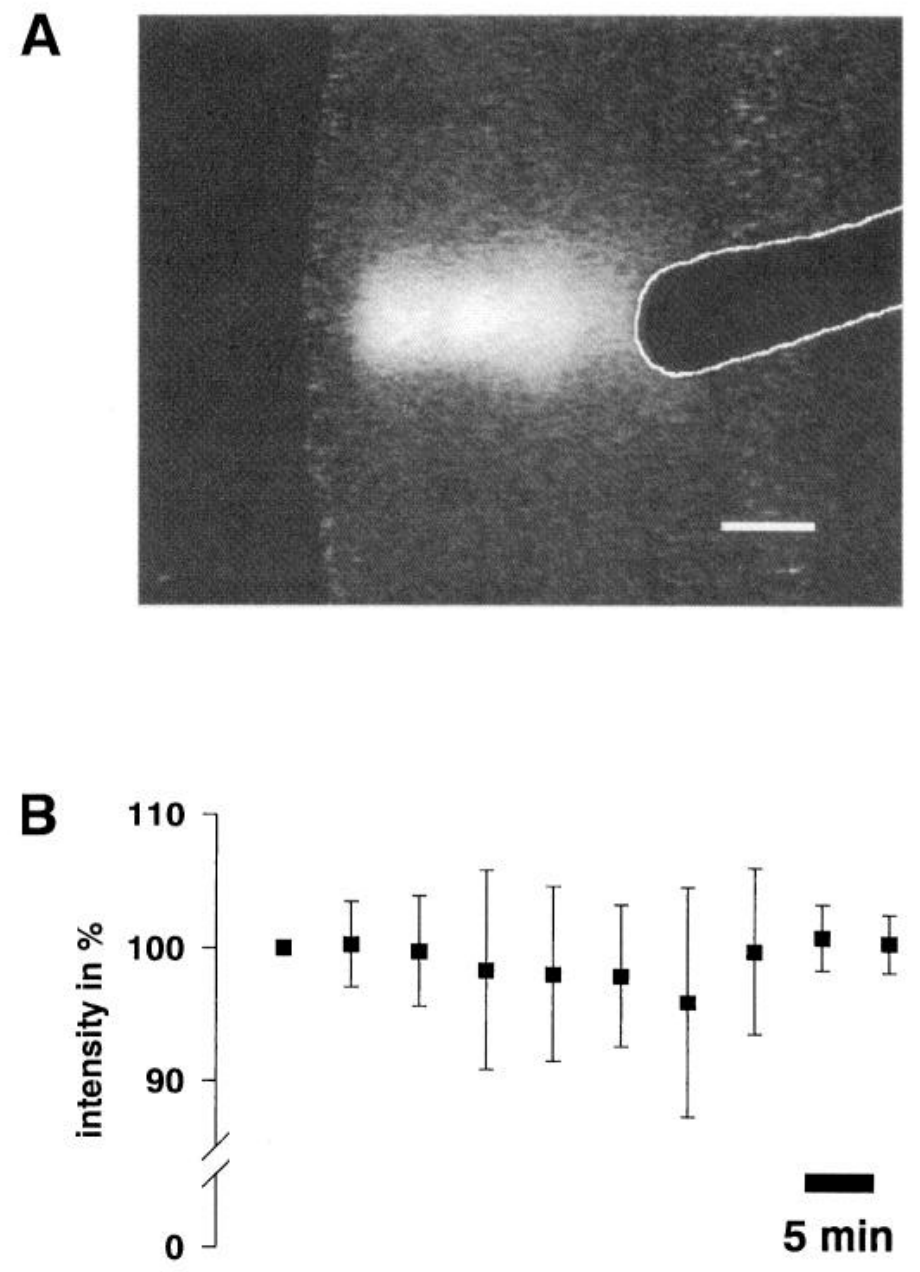

Figure 1. Reproducibility of activity-dependent change in IOS in rat neocortical slice. $A$, IOS at peak intensity $3 \mathrm{sec}$ after onset of stimulation. Stimulation electrode is marked by a white boundary. Note that the size of the stimulation electrode is overestimated because the tip is bent $\sim 90$ degrees and positioned perpendicular to the surface of the slice. Scale bar, $300 \mu \mathrm{m}$. B, Reproducibility of the peak amplitudes of IOS. Peak amplitudes of 10 subsequent stimulations with time intervals of 5 min were standardized to the first signal, which was set to $100 \%$. Data show means and SDs of five experiments.

transmission was blocked, and we failed to induce IOSs by afferent stimulation.

To quantify the reproducibility of IOSs, 10 subsequent stimulation-induced IOSs with time intervals of $5 \mathrm{~min}$ were recorded. Peak amplitudes of IOSs were evaluated and standardized to the first evoked signal, which was set to $100 \%$ (Fig. $1 B$ ). The mean amplitude of the IOSs showed a high reproducibility. The greatest deviation of mean IOSs in the signal series was $4.1 \pm$ $8.6 \%(n=5)$.

In a subsequent series of experiments, the stimulus-induced time courses of IOSs were compared with the time courses of changes in extracellular $\mathrm{K}^{+}$concentration in layer II/III. Both parameters were determined simultaneously. The experiments showed that the IOSs were accompanied by significant changes of extracellular $\mathrm{K}^{+}$concentration. Peak levels up to $10 \mathrm{~mm}$ of $\mathrm{K}^{+}$ were encountered. Changes in extracellular $\mathrm{K}^{+}$concentration were generally faster than the IOSs (Fig. $2 A$ ). To characterize the time courses, time-to-peak and the time constant of signal decay were evaluated. The IOSs reached their maximum amplitudes of $3.8 \pm 0.6 \mathrm{sec}(n=20)$ after onset of stimulation. The signals 
A

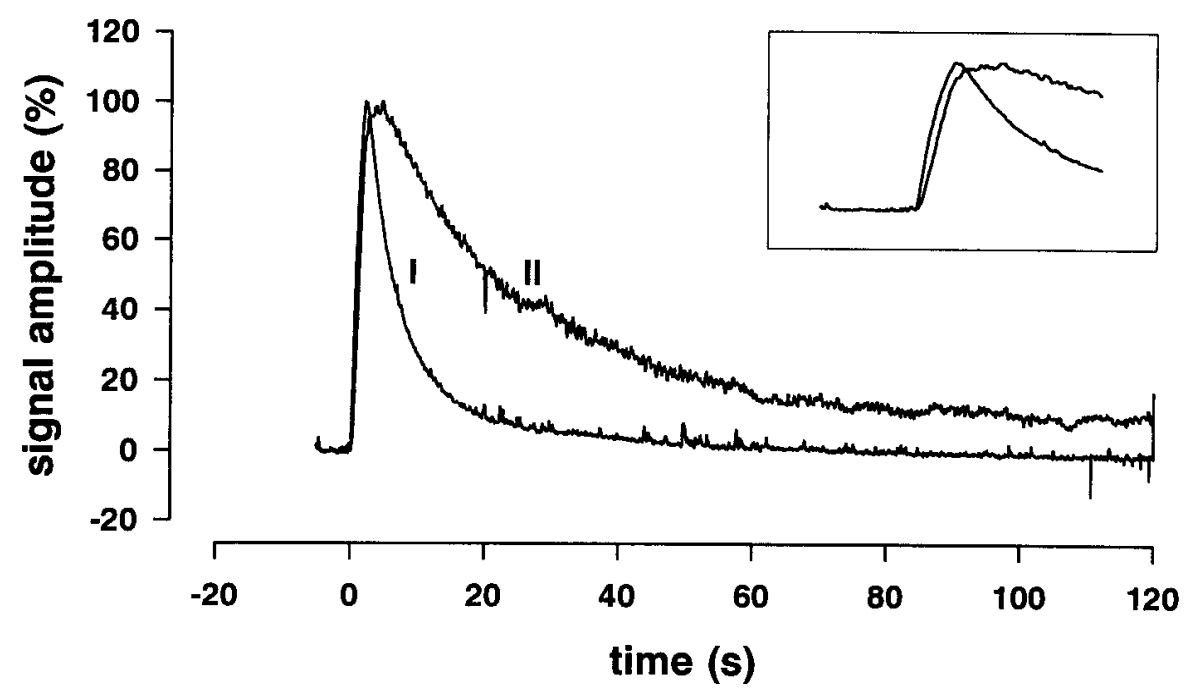

Figure 2. Comparison between the time courses of stimulus-induced changes in IOS, and extracellular potassium and TMA concentration after afferent stimulation. Peak amplitudes are standardized to $100 \%$. Onset of stimulation at $t=0 \mathrm{sec}$. $A$, Time courses of changes in extracellular potassium concentration (I) and IOS (II) in layer II/III. Resting potassium concentration $3.0 \mathrm{~mm}$; peak (100\%) $6.8 \mathrm{~mm}$. Inset shows the first $15 \mathrm{sec}$ on a faster time scale. $B$, Time courses of changes in TMA concentration (I) and IOS (II) in layer II/III. Because TMA is not membrane-permeable, its change indicates alterations of ECS volume. Resting TMA concentration $10.0 \mathrm{mM}$; peak $10.3 \mathrm{mM}$, indicating a change of ECS volume by $3 \%$. Inset shows the first $15 \mathrm{sec}$ on a faster time scale.

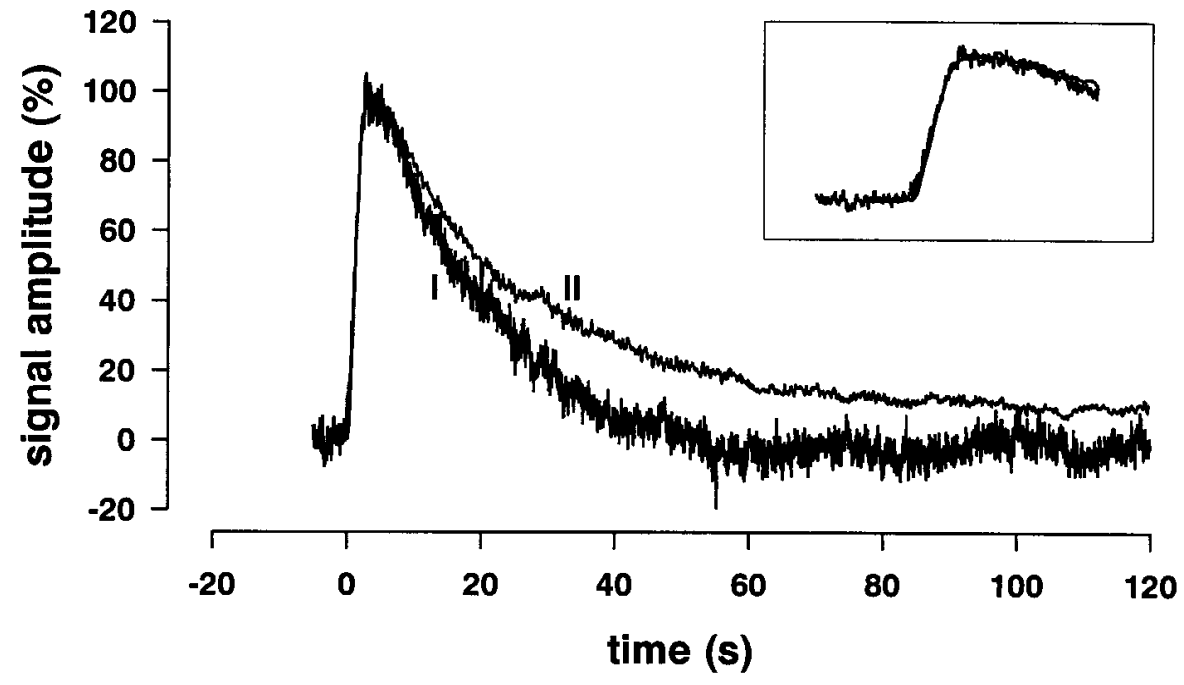

decayed with a monoexponential time course and a mean time constant of $40 \pm 10 \mathrm{sec}(n=20)$. At corresponding locations, we observed a transient increase in extracellular $\mathrm{K}^{+}$concentration after afferent stimulation (Fig. 2A). Before stimulation, a mean extracellular $\mathrm{K}^{+}$concentration of $3.0 \pm 0.1 \mathrm{mM}(n=10)$ was measured. After onset of stimulation, the extracellular $\mathrm{K}^{+}$concentration rose $2.1 \pm 0.04 \mathrm{sec}(n=10)$ up to maximum values of $7.4 \pm 0.9 \mathrm{mM}(n=10)$. After end of stimulation, the $\mathrm{K}^{+}$concentration decayed with a monoexponential time course and a mean time constant of $3.1 \pm 0.6 \mathrm{sec}(n=10)$. The speed of increases in extracellular $\mathrm{K}^{+}$concentration was higher than that of the corresponding IOSs ( $p<0.01$, paired Student's $t$ test). Furthermore, the mean time constant of extracellular $\mathrm{K}^{+}$concentration decay after end of stimulation was considerably smaller than the mean time constant of IOS decay ( $p<0.001$, paired Student's $t$ test).

For measurement of changes in ECS volume, the preparations were superfused with aCSF containing $10 \mathrm{mM}$ TMA-Cl. In TMA-

containing solutions, the IOS and the field potential did not show any detectable changes compared with control solutions. After afferent stimulation, a transient increase of TMA concentration in layer II/III could be detected (Fig. $2 B$ ). This increase indicates a shrinkage of ECS volume. Assuming that no TMA enters the cells and that it does not diffuse away from the area with increased concentration, the change of ECS volume can be calculated from the TMA concentration. In this series of experiments, the mean ECS volume shrinkage, evaluated from changes in TMA concentration, amounted to $3.5 \pm$ $1.4 \%(n=10)$. The TMA signals reached their maximum $3.1 \pm 0.3$ $\sec (n=10)$ after onset of stimulation and decayed with a monoexponential time course and a mean time constant of $14 \pm 4.7 \mathrm{sec}(n=$ 10). The mean time interval in which the TMA signals reached their maximum amplitudes was greater than the mean time-to-peak of extracellular $\mathbf{K}^{+}$concentration increases ( $p<0.01$, Student's $t$ test). There was no significant difference between time-to-peak of TMA concentration increases and corresponding IOSs. However, the 
mean time constant of TMA concentration decay to control levels after stimulation-induced shrinkage was smaller than the mean time constant of the corresponding IOS decay $(p<0.01$, paired Student's $t$ test).

There is some evidence that glial cell swelling mediated by net $\mathrm{KCl}$ uptake, via a furosemide-sensitive $\mathrm{Na}-\mathrm{K}-2 \mathrm{Cl}$ cotransport, is responsible for changes in ECS volume during neuronal activity (Ransom et al., 1985). We therefore tested whether the IOSs and the changes of ECS volume could be influenced by a reduction of extracellular $\mathrm{Cl}^{-}$concentration or by perfusion with furosemide. All data were standardized to control values.

Reduction of extracellular $\mathrm{Cl}^{-}$concentration to $17 \mathrm{~mm}$ by equimolar substitution with gluconate reversibly diminished peak amplitudes of IOSs (Figs. 3, 4) to $7 \pm 9 \%(n=10)$. After wash with standard aCSF, the peak amplitudes of IOSs increased again to $83 \pm 9 \%(n=$ $10)$. There was a dissociation between the behavior of the IOSs and the changes in extracellular $\mathrm{K}^{+}$concentration. Whereas the former disappeared completely in low $\mathrm{Cl}^{-}$solution, the latter were only slightly reduced to $80 \pm 10 \%$ ( $n=5$; Fig. 3 ). The time course of the $\mathrm{K}^{+}$signals was considerably prolonged under these conditions. In solutions with lowered extracellular $\mathrm{Cl}^{-}$concentration, the extracellular $\mathrm{K}^{+}$concentration reached maximum amplitudes of $2.1 \perp 0.03$ $\sec (n=5)$ after onset of stimulation, and decayed monoexponentially with a time constant of $4.1+0.5 \sec (n=5)$. The time-to-peak was not different from control, but the time constant of decay was larger than values under control conditions $(p<0.01$, paired Student's $t$ test). The peak of TMA increases was reduced to $12 \pm 3 \%$ $(n=5)$ by perfusion with low $\mathrm{Cl}^{-}$aCSF (Fig. 4). This reduction was not significantly different from the reduction of corresponding IOSs, but differed from alterations in extracellular $\mathrm{K}^{+}$concentration $(p<$ 0.01 , Student's $t$ test).

Perfusion of the slice preparation with furosemide had similar effects on IOSs as did perfusion with low chloride solution. Perfusion with $5 \mathrm{~mm}$ furosemide for $20 \mathrm{~min}$ reversibly reduced peak amplitudes of IOSs to $11 \pm 7 \%$ ( $n=10$; Figs. 5, 6). They reached $79 \pm 14 \%$ of control level after washout. Peak amplitudes of extracellular $\mathbf{K}^{+}$ concentration under the same conditions were reduced to $42 \pm 6 \%$ ( $n-5$; Fig. 5). This reduction was smaller than the reduction of the corresponding IOSs ( $p<0.01$, paired Student's $t$ test). After $20 \mathrm{~min}$ of perfusion with $5 \mathrm{~mm}$ furosemide, afferent stimulations resulted in an increase of extracellular $\mathrm{K}^{+}$concentration, which reached maximum amplitudes $2.1 \pm 0.1 \mathrm{sec}(n=5)$ after onset of stimulation and decayed monoexponentially with a time constant of $4.7 \pm 1.0 \mathrm{sec}(n$ $=5$ ). Time-to-peak of potassium increase was not significantly different from control conditions, but signal decay was prolonged $(p<$ 0.02 , paired Student's $t$ test). After washout for at least $60 \mathrm{~min}$, the peak amplitudes of extracellular $\mathrm{K}^{+}$concentration recovered to $81 \pm$ $15 \%(n=5)$ of control values. Furosemide caused changes of ECS volume shrinkage, which were similar to the changes of IOSs (Fig. 6). After treatment with $5 \mathrm{~mm}$ furosemide for $20 \mathrm{~min}$ and afferent stimulation, the TMA signals were decreased to $15 \pm 6 \%(n=5)$. This signal reduction was not significantly different from the corresponding reduction of the amplitudes of IOSs. After washout, peak amplitudes of ECS volume shrinkage reached $99 \pm 6 \%(n=5)$ of control values.

\section{DISCUSSION}

The size of the columnar-shaped, activity-dependent IOSs in rat neocortical slices corresponds to the extent of electrical activity (Holthoff et al., 1994). With other techniques, such as voltagesensitive dyes or $\left[{ }^{14} \mathrm{C}\right]$ deoxyglucose autoradiography, similar patterns of activity were described in guinea pig neocortex and rat barrel cortex (Kossut and Hand, 1984; Albowitz and Kuhnt, 1993). Except for very strong stimuli, which cause a diffuse widening of the response area, the size of the columns does not correspond to stimulation strength, suggesting that the columns seen with this technique may correlate to the functional architecture of these brain areas (Holthofl el al., 1994). Advantages of IOSs in detecting neuronal activity are their good reproducibility and the absence of pharmacological side effects and photodynamic damages (Grinvald et al., 1982) (for review, see Grinvald et al., 1988).

Activity-dependent IOSs in rat neocortical slices have a slow time course, which exceeds the time course of electrical activity (Holthoff et al., 1994). By measuring changes of light transmission in rat hippocampal slices, MacVicar and Hochman (1991) found similar time courses of IOSs. In chicken retina, one component of IOS, accompanied by spreading depression, was supposed to be correlated to glial depolarization (Fernandes de Lima et al., 1994). Because glial cells conventionally behave like $\mathrm{K}^{+}$electrodes (Kettenmann et al., 1983), the time course of extracellular $\mathrm{K}^{+}$concentration should be a good measure of their behavior. In this study, the time courses of IOSs and extracellular $\mathrm{K}^{+}$increases were not correlated.

Our data suggest that activity-dependent IOSs in rat neocortical slices reveal changes in ECS volume. It could not be determined, however, whether the optical signal derived from swollen cells or from a decrease in ECS volume. The simultaneous measurement of stimulation-induced IOSs and the concomitant changes in extracellular space yielded similar time courses of both parameters. However, there are small deviations between the time courses in the phase of signal decay, which seem to be attributable to the technique of measuring changes in extracellular space used in this study. Evaluation of the size of extracellular space is possible with a technique based on a diffusion analysis of an extracellular marker (Nicholson, 1992; Lehmenkühler et al., 1993). The volume fraction and tortuosity of the tissue can be evaluated between two electrodes. Unfortunately, the time resolution of this technique is only in the range of several tens of seconds. In the present study, the activity-induced shrinkage of ECS volume was measured by monituring the concentration of the extracellular marker TMA, which was superfused over the preparation. This technique allows for the determination of quantitative changes in ECS volume with high resolution in time (Hansen and Olsen, 1980), because TMA is mostly restricted to ECS (Nicholson, 1992). There are two limitations in using the extracellular marker TMA to measure alterations of ECS volume. First, TMA may be taken up by glial cells (Ballanyi et al., 1990). This process appears to reach a steady state rapidly after TMA exposure, suggesting that the uptake mechanism is immediately saturated and, thus, did not cause any major error in our measurements. Second, in case of slow focal changes of ECS, the technique will not monitor the ECS correctly because TMA may diffuse away from the area with a decrease of ECS volume to the surrounding brain and bath solution. In accordance with these expectations, the time course of IOS and the time course of TMA concentration were identical in the fast phase of signal increase, whereas in the slow phase of decay, the TMA signal decreased with a smaller time constant

The experiments with low chloride solutions and furosemide support the assumption that activity-dependent IOSs in rat neocortical slices are attributable to changes in ECS volume. Our data confirm previous studies in which IOSs were blocked by perfusion with furosemide or low chloride solutions, which are conditions known to prevent activity-induced changes in ECS volume (Ran- 

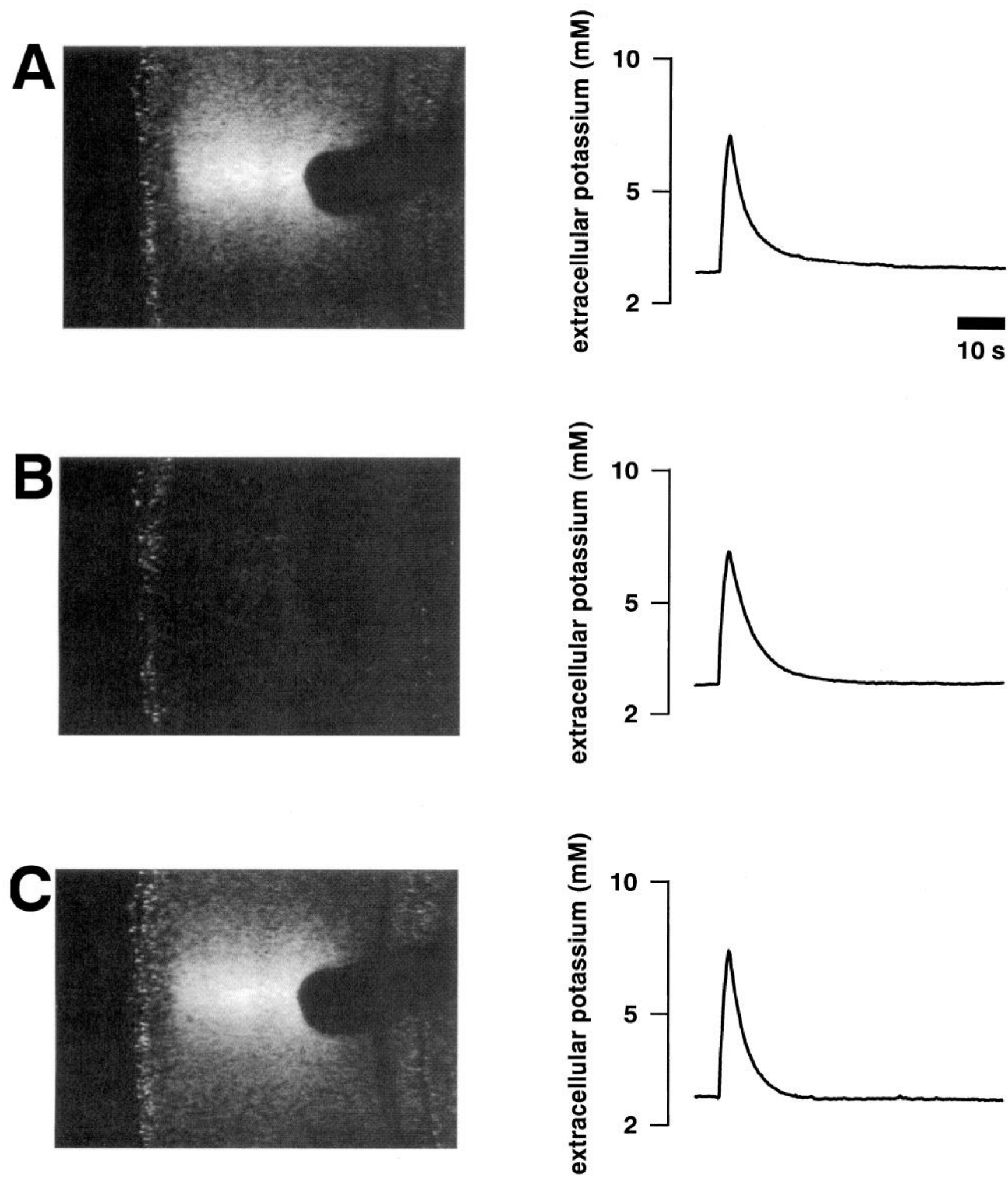

Figure 3. Alteration of IOS and potassium transients by low chloride solution. $A$, Afferent stimulation for 2 sec caused a columnar-shaped IOS and a transient increase of extracellular potassium concentration. Original figure on left was obtained $3 \mathrm{sec}$ after onset of stimulation. $B$, Reduction of extracellular $\mathrm{Cl}^{-}$concentration to $17 \mathrm{~mm}$ by substitution with equimolar gluconate caused a decrease of IOS peak amplitude. Extracellular potassium increase was reduced only slightly. Note increase of decay time in $B$ compared with $A$ or $C$. $C$, Recovery under control conditions was complete.

som et al., 1985; MacVicar and Hochman, 1991). Additionally, IOSs and changes in ECS volume were reduced similarly under these conditions, whereas the effect of these treatments on changes of extracellular $\mathrm{K}^{+}$concentration did not correspond to changes in IOSs.
A swelling of cells and the associated changes of ECS are well known effects of neuronal activity. A long-lasting decrease in the ECS volume has been observed after repetitive stimulation of rat spinal cord (Svoboda and Sykova, 1991). This decrease in ECS volume was accompanied by an increase in extracellular potas- 

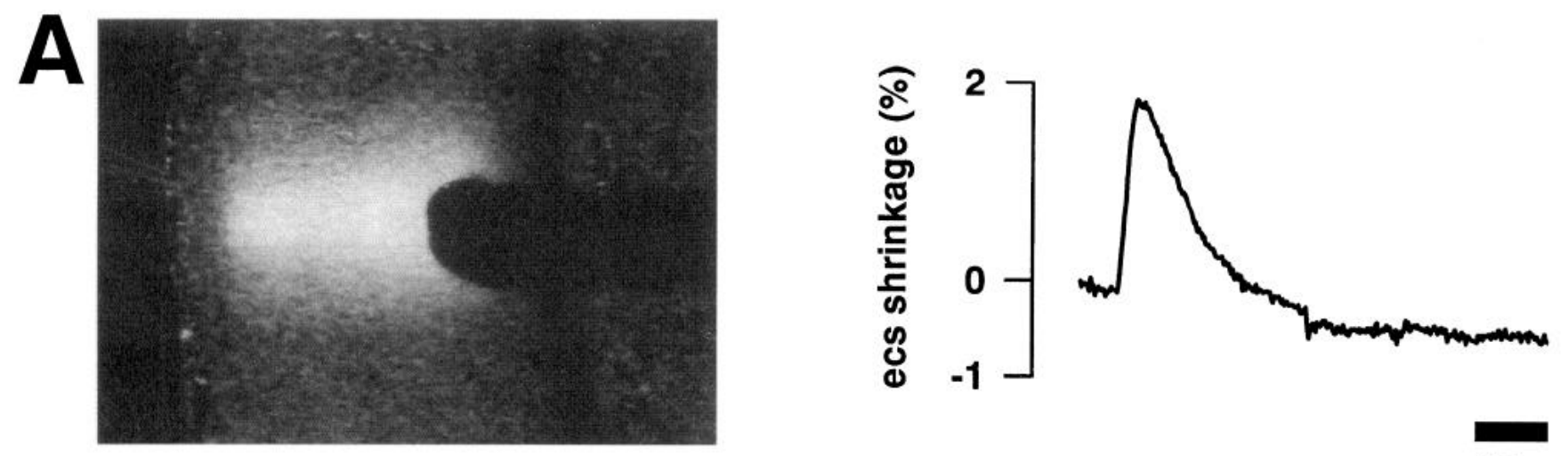

$10 \mathrm{~s}$
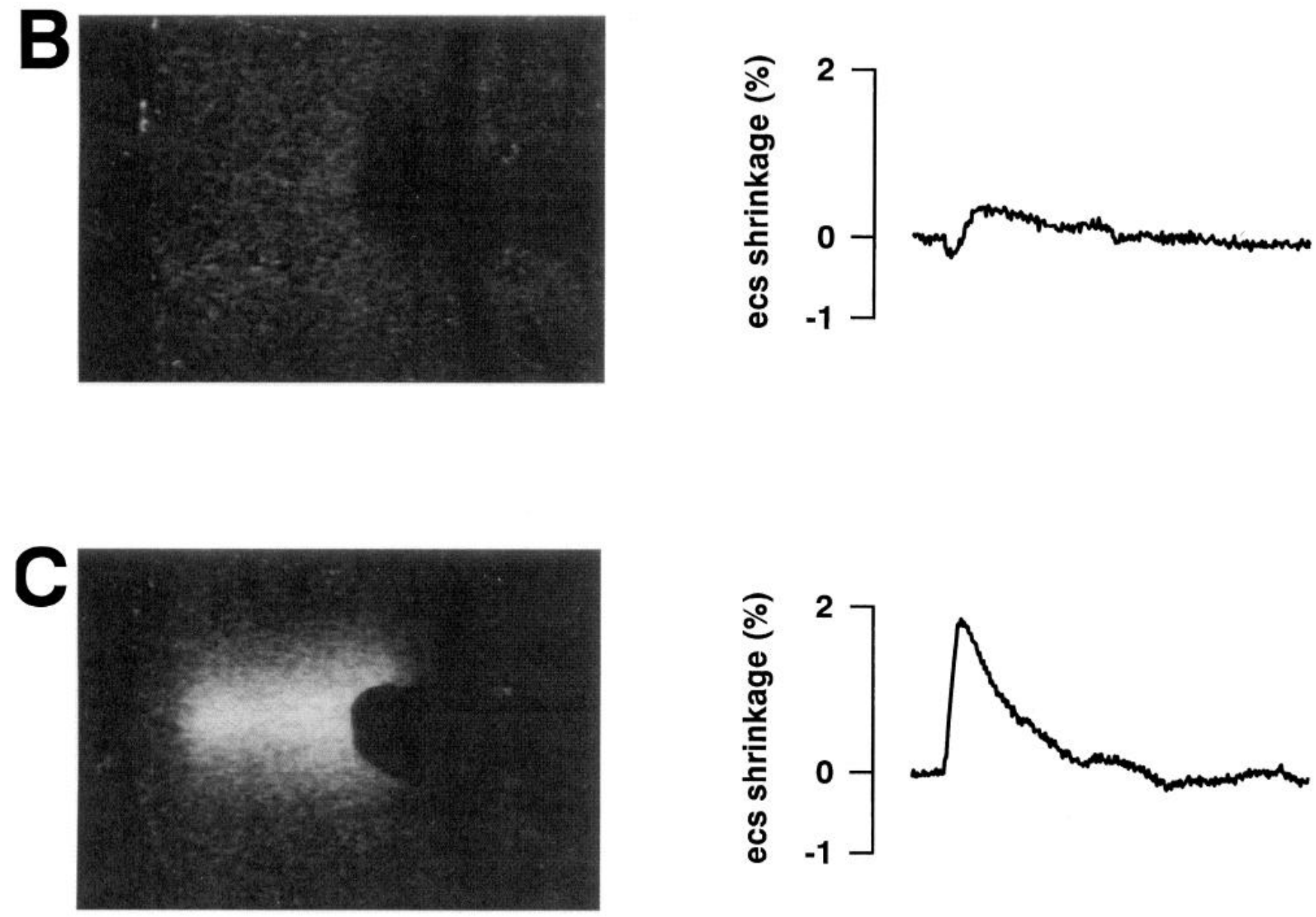

Figure 4. Alteration of IOS and TMA signal in low chloride solution. A, Afferent stimulation for 2 sec caused a columnar-shaped IOS and a transient increase of TMA signal. Original figure on left was obtained $3 \mathrm{sec}$ after onset of stimulation. $B$, Reduction of extracellular $\mathrm{Cl}^{-}$concentration to $17 \mathrm{mM}$ by substitution with equimolar gluconate diminished activity-dependent IOS and the accompanied TMA signal. $C$, Recovery under control conditions was complete.

sium concentration. In vivo stimulation of the cortical surface in cats induces elevations of extracellular potassium concentration, which are also accompanied by a reduction in ECS volume (Dietzel et al., 1980). An increase in ECS volume has been described in frog retina after stimulation with light (Huang and Karwoski, 1992). This increase is associated with a decrease of extracellular $\mathrm{K}^{+}$concentration. These observations, as well as the present data, indicate that the degree, although not the time course, of changes in extracellular $\mathrm{K}^{+}$concentration parallel the activity-dependent changes in ECS volume.

Activity-dependent shrinkage in ECS volume can be attributable to a swelling of neurons and/or glial cells. A swelling of neurons during activity may be attributable to $\mathrm{NaCl}$ influx or to enhanced metabolic processes, which increase intracellular osmotic pressure (Dietzel et al., 1989). There is little experimental data about activity-induced changes of neuronal cell volume. In 

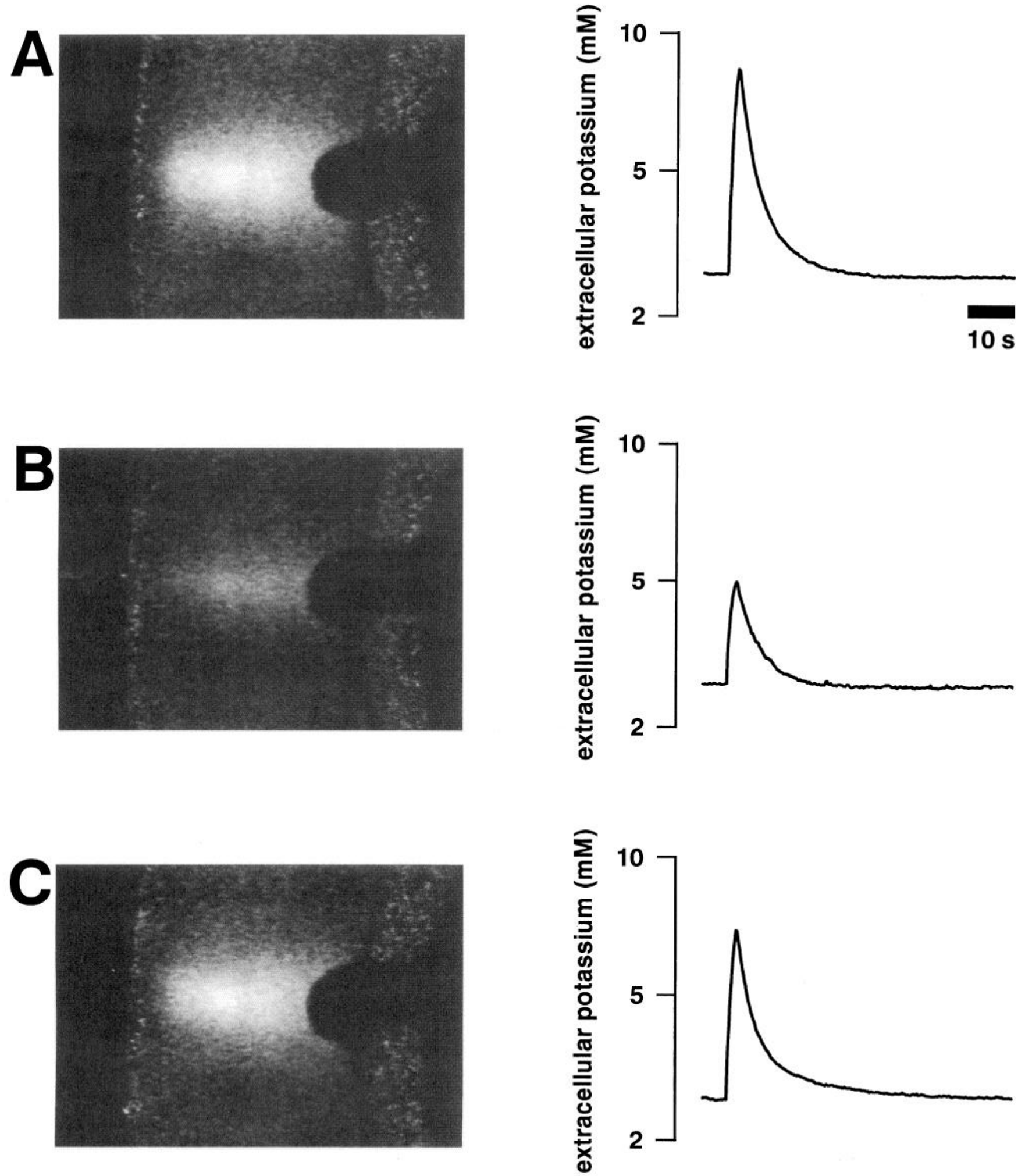

Figure 5. Alteration of IOS and potassium transients by furosemide. A, Afferent stimulation for 2 sec caused a columnar-shaped IOS and a transient increase of extracellular potassium concentration. Original figure on left was obtained $3 \mathrm{sec}$ after onset of stimulation. $B$, Perfusion with 5 mm furosemide for $20 \mathrm{~min}$ caused a pronounced decrease in IOS peak amplitude, but only a moderate reduction of potassium peak amplitude. $C$, Recovery under control conditions was nearly complete.

rat spinal motor neurons, Serve et al. (1988) described a shrinkage of neuronal somata after repetitive electrical stimulation. Nevertheless, during equal conditions a shrinkage of ECS occurs.

A swelling of cells also has been postulated in the context of potassium clearance mechanisms (which occur after strong neuronal excitation) and the concomitant increase in extracellular potassium. There is substantial evidence implying that glial cells are prominently involved in potassium-induced changes of ECS 

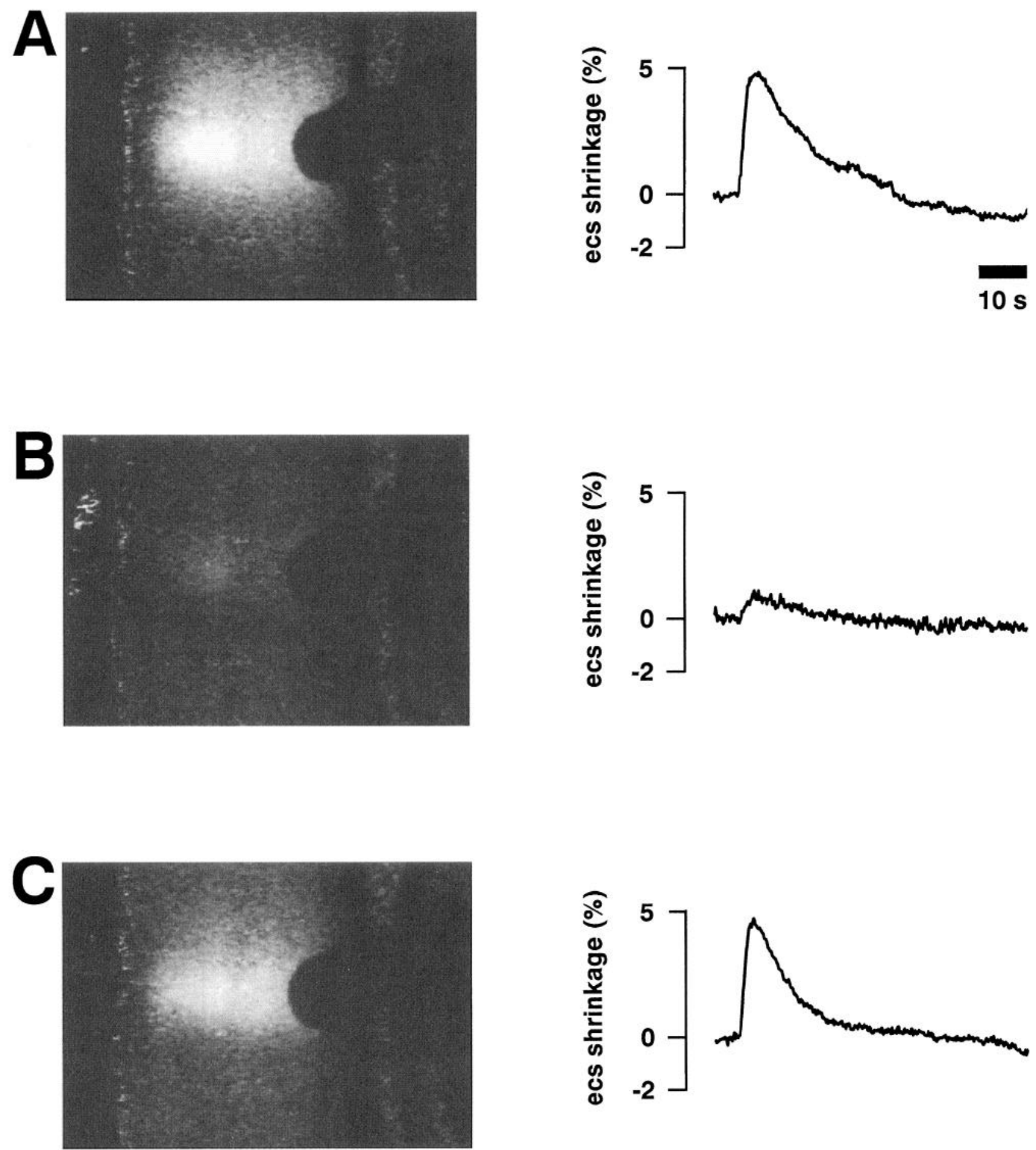

Figure 6. Alteration of IOS and TMA signal by furosemide. A, Afferent stimulation for 2 sec caused a columnar-shaped IOS and a transient increase of TMA signal. $B$, Perfusion with $5 \mathrm{~mm}$ furosemide for $20 \mathrm{~min}$ caused a pronounced decrease in IOS peak amplitude and a similar reduction of TMA signal. $C$, Recovery under control conditions was nearly complete.

volume (Ransom et al., 1985). At least four different mechanisms have been described regarding how glial cells may take up potassium from the ECS (for review, see Kimelberg et al., 1993). First, an uptake of extracellular $\mathrm{K}^{+}$via the $\mathrm{Na}^{+}-\mathrm{K}^{+}$pump may occur with $\mathrm{Cl}^{-}$accompanying $\mathrm{Na}^{+}$to preserve charge neutrality. This mechanism would lead to cell shrinkage and therefore have an opposite osmotic effect to that of the activity-induced ECS volume shrinkage, which is caused by cell swelling. Second, there may be an uptake of $\mathrm{K}^{+}$and $\mathrm{Cl}^{-}$through independent channels driven by the depolarization attributable to increased extracellular $\mathrm{K}^{+}$concentration. It is called the Donnan depolarization-driven uptake of $\mathrm{KCl}$. This uptake should be independent of treatment with furosemide, although furosemide can have nonspecific blocking effects on chloride channels in addition to its effects as a transport inhibitor. A possible third mechanism is the so-called spatial potassium buffer mechanism, first described by Orkand et al. 

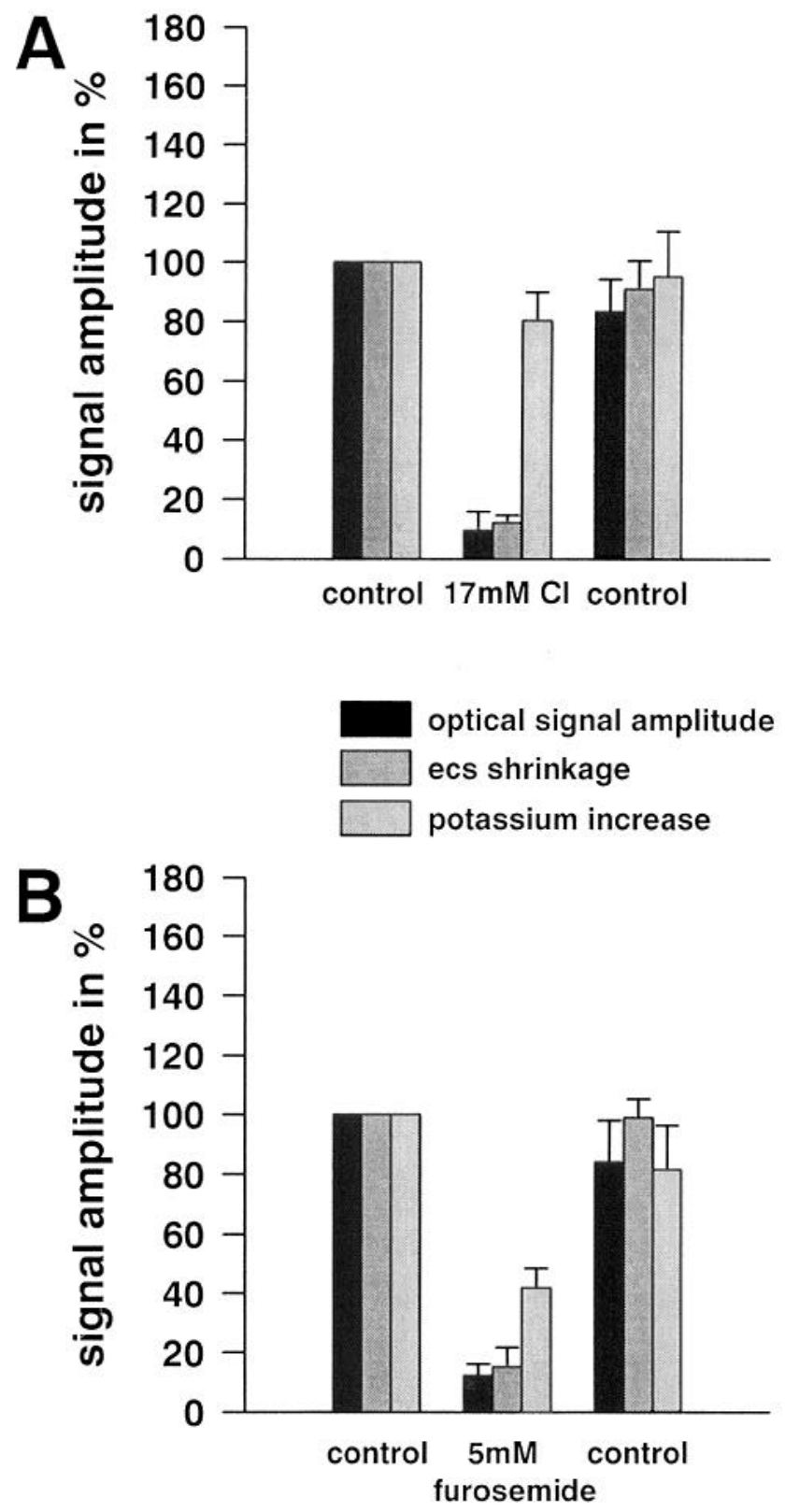

Figure 7. Quantitative evaluation of low chloride effects and furosemide effects on amplitudes of stimulus-induced IOS, ECS volume shrinkage, and extracellular potassium concentration increase. All data denote means and SDs of five experiments. $A$, Influence of reduction extracellular $\mathrm{Cl}^{-}$ concentration to $17 \mathrm{~mm}$ by substitution with equimolar gluconate on peak amplitude of IOS, ECS volume shrinkage, and extracellular potassium concentration increase. $B$, Influence of treatment with $5 \mathrm{~mm}$ furosemide for $20 \mathrm{~min}$ on peak amplitude of IOS, ECS volume shrinkage, and extracellular potassium concentration increase.

(1966). Because glial cells are electrotonically coupled, they may take up potassium at the site where it is expelled from the neurons, liberating it more distantly, and thus acting as a spatial potassium buffer. Such a mechanism would lead to a redistribution of ions in the extracellular and intracellular compartments and a concomitant cell swelling at the site of maximal $\mathrm{K}^{+}$elevation (Dietzel et al., 1980). This mechanism should not be influenced by variations of the extracellular $\mathrm{Cl}^{-}$concentration. However, the present study showed that in low $\mathrm{Cl}^{-}$solutions it was possible to diminish both activity-dependent IOSs and changes in ECS volume almost completely. Thus, our data indicate that such a mechanism, although it may be present, is probably not the major determinant of activity-induced shrinkage in ECS volume under the present conditions.

The fourth mechanism of potassium clearance may be that of an uptake of $\mathrm{KCl}$ into glial cells mediated by a furosemidesensitive Na-K-2Cl cotransporter (Walz and Hertz, 1984; Walz and Hinks, 1986). In our study, it was possible to diminish both stimulation-induced IOS and ECS volume shrinkage by equal amounts by lowering extracellular $\mathrm{Cl}^{-}$concentration (Fig. $7 A$ ). The extracellular $\mathrm{K}^{+}$concentration increase was still present, indicating that stimulation-induced neuronal activity was not altered greatly. Compared with control conditions, the prolonged decay indicates an impaired mechanism of $\mathrm{K}^{+}$clearance. It has been reported that astrocytes equilibrated at $3 \mathrm{~mm}$ $\mathrm{K}^{+}$and suddenly exposed to $12 \mathrm{~mm} \mathrm{~K}^{+}$display a net $\mathrm{K}^{+}$uptake accompanied by cell swelling (Walz and Hinks, 1985). Although under these conditions the furosemide-sensitive part of glial $\mathrm{K}^{+}$uptake is only small (Walz and Hertz, 1984), the accompanied cell swelling was supposed to be attributable to $\mathrm{KCl}$ uptake via a $\mathrm{Na}-\mathrm{K}-2 \mathrm{Cl}$ cotransport and a concomitant water influx (Walz and Hinks, 1986). This assumption is supported by the observation that astrocyte swelling can be inhibited by the $\mathrm{Na}-\mathrm{K}-2 \mathrm{Cl}$ cotransport blocker furosemide or by reduction of extracellular $\mathrm{Cl}^{-}$concentration (Walz and Hinks, 1985). In accordance with this observation, in our study treatment with furosemide reduced both IOS and ECS volume shrinkage by equal amounts (Fig. $7 B$ ).

The present data suggest that IOSs do not monitor alterations of extracellular $\mathrm{K}^{+}$concentration, but instead correspond to changes in ECS volume. We suggest that the IOSs are attributable to a furosemide-sensitive uptake of $\mathrm{KCl}$ in glial cells and a concomitant water influx. Monitoring of IOSs may provide a sensitive means to study changes in ECS volume, which occur in clinical conditions such as ischemic infarction and epilepsy.

\section{REFERENCES}

Albowitz B, Kuhnt U (1993) The contribution of intracortical connections to horizontal spread of activity in the neocortex as revealed by voltage sensitive dyes and a fast optical recording method. Eur J Neurosci 5:1349-1359.

Andrew RD, MacVicar BA (1994) Imaging cell volume changes and neuronal excitation in the hippocampal slice. Neuroscience 62:371-383.

Ballanyi K, Grafe P, Serve G, Schlue WR (1990) Electrophysiological measurements of volume changes in leech neuropile glial cells. Glia 3:151-158.

Dietzel I, Heinemann U, Hofmeier G, Lux HD (1980) Transient changes in the size of the extracellular space in the sensorimotor cortex of cats in relation to stimulus-induced changes in potassium concentration. Exp Brain Res 40:432-439.

Dietzel I, Heinemann U, Lux HD (1989) Relations between slow extracellular potential changes, glial potassium buffering, and electrolyte and cellular volume changes during neuronal hyperactivity in cat brain. Glia 2:25-44.

Federico P, Borg SG, Salkauskus AG, MacVicar BA (1994) Mapping patterns of neuronal activity and seizure propagation by imaging intrinsic optical signals in the isolated whole brain of the guinea-pig. Neuroscience 58:461-480.

Fernandes de Lima VM, Goldermann M, Hanke WRL (1994) Calcium waves in gray matter are due to voltage-sensitive glial membrane channels. Brain Res 663:77-83.

Frostig RD, Lieke EE, Ts'o DY, Grinvald A (1990) Cortical functional architecture and local coupling between neuronal activity and the microcirculation revealed by in vivo high resolution optical imaging of intrinsic signals. Proc Natl Acad Sci USA 87:6082-6086.

Gochin PM, Bedenbaugh P, Gelfand JJ, Gross CG, Gerstein GL (1992) Intrinsic signal optical imaging in the forepaw area of rat somatosensory cortex. Proc Natl Acad Sci USA 89:8381-8383. 
Grinvald A, Hildesheim R, Farber IC, Anglister L (1982) Improved fluorescent probes for the measurement of rapid changes in membrane potential. Biophys J 39:301-308.

Grinvald A, Lieke E, Frostig RD, Gilbert CD, Wiesel TN (1986) Functional architecture of cortex revealed by optical imaging of intrinsic signals. Nature 324:361-364.

Grinvald A, Frostig RD, Lieke E, Hildesheim R (1988) Optical imaging of neuronal activity. Physiol Rev 68:1285-1366.

Haglund MM, Ojemann GA, Hochman DW (1992) Optical imaging of epileptiform and functional activity in human cerebral cortex. Nature 358:668-671.

Hansen AJ, Olsen CE (1980) Brain extracellular space during spreading depression and ischemia. Acta Physiol Scand 108:355-365.

Hill DK, Keynes RD (1949) Opacity changes in stimulated nerve. J Physiol 108:278-281.

Holthoff K, Dodt HU, Witte OW (1994) Changes in intrinsic optical signal of rat neocortical slices following afferent stimulation. Neurosci Lett 180:227-230.

Huang B, Karwoski CJ (1992) Light-evoked expansion of subretinal space volume in the retina of the frog. J Neurosci 12:4243-4252.

Kettenmann H, Sonnhof U, Schachner M (1983) Exclusive potassium dependence of the membrane potential in cultured mouse oligodendrocytes. J Neurosci 3:500-505.

Kimelberg HK, Jalonen T, Walz W (1993) Regulation of the brain microenvironment: transmitters and ions. In: Astrocytes: pharmacology and function (Murphy S, ed), pp 193-228. New York:Academic.

Kossut M, Hand P (1984) Early development of changes in cortical representation of $\mathrm{C} 3$ vibrissa following neonatal denervation of surrounding vibrissa receptors: a 2-deoxyglucose study in the rat. Neurosci Lett 46:7-12.

Lehmenkühler A, Sykova E, Svoboda J, Zilles K, Nicholson C (1993) Extracellular space parameters in the rat neocortex and subcortical white matter during postnatal development determined by diffusion analysis. Neuroscience 55:339-351.
Lipton P (1973) Effects of membrane depolarization on light scattering by cerebral cortical slices. J Physiol (Lond) 231:365-383.

MacVicar BA, Hochman D (1991) Imaging of synaptically evoked intrinsic optical signals in hippocampal slices. J Neurosci 11:1458-1469.

McManus M, Fischbarg J, Sun A, Hebert S, Strange K (1993) Laser light-scattering system for studying cell volume regulation and membrane transport processes. Am J Phys 265:C562-C570.

Nicholson C (1992) Quantitative analysis of extracellular space using the method of TMA + iontophoresis and the issue of TM $\Lambda+$ uptake. Can J Physiol Pharmacol 70:S314-S322.

Nicholson C, Phillips JM (1981) Ion diffusion modified by tortuosity and volume fraction in the extracellular micruenvirunment of the rat cerebellum. J Physiol (Lond) 321:225-257.

Orkand RK, Nicholls JG, Kuffler SW (1966) Effect of nerve impulses on the membrane potential of glial cells in the central nervous system of amphibia. J Neurophysiol 29:788-806.

Ransom BR, Yamate CL, Connors BW (1985) Activity-dependent shrinkage of extracellular space in rat optic nerve: a developmental study. J Neurosci 5:532-535.

Serve G, Endres W, Grafe P (1988) Continuous electrophysiological measurements of changes in cell volume of motoneurons in the isolated frog spinal cord. Pflügers Arch 411:410-415.

Svoboda J, Sykova E (1991) Extracellular space volume changes in the rat spinal cord produced by nerve stimulation and peripheral injury. Brain Res 560:216-224.

Walz W, Herk L (1984) Intense furosemide-sensitive potassium accumulation in astrocytes in the presence of pathologically high extracellular potassium levels. J Cereb Blood Flow Metab 4:301-304.

Walz W, Hinks EC (1985) Carrier-mediated $\mathrm{KCl}$ accumulation accompanied by water movements is involved in the control of physiological $\mathrm{K}^{+}$levels by astrocytes. Brain Res 343:44-51.

Walz W, Hinks EC (1986) A transmembrane sodium cycle in astrocytes. Brain Res 368:226-232. 